\title{
Why Bystanders Did Not Perform Cardiopulmonary Resuscitation on Out-of-Hospital Cardiac Arrest Patients: A Multi-Center Study in Hanoi (Vietnam)
}

\author{
Dinh Hung Vu, MD; ${ }^{1}$ Bui Hai Hoang, MD, PhD; ${ }^{1,2}$ (i) Ngoc Son Do, MD, PhD; ${ }^{3}$ Giang Phuc Do, MD; ${ }^{1}$ \\ Xuan Dung Dao, MD; ${ }^{4}$ Huu Huan Nguyen, MD; ${ }^{5}$ Quang Thuy Luu, MD; ${ }^{6}$ Lan Hieu Nguyen, MD, \\ $\mathrm{PhD} ;{ }^{1,2}$ Shinji Nakahara, MD, MS, $\mathrm{PhD}^{7}$ (i)
}

1. Hanoi Medical University Hospital, Hanoi, Vietnam

2. Hanoi Medical University, Hanoi, Vietnam

3. Bach Mai Hospital, Hanoi, Vietnam

4. Hanoi Heart Hospital, Hanoi, Vietnam

5. Ministry of Agriculture Hospital, Hanoi, Vietnam

6. Vietduc Hospital, Hanoi, Vietnam

7. Graduate School of Health Innovation, Kanagawa University of Human Services, Kawasaki, Japan

\section{Correspondence: \\ Associate Professor Hoang Bui Hai \\ Hanoi Medical University \\ 1 Ton That Tung St, Dong Da, Ha Noi, Viet Nam \\ Email: hoangbuihai@hmu.edu.vn}

Conflicts of interest/funding: This study was supported by the Japan Society for the Promotion of Science KAKENHI (16K11422 and 19K09403); the funding body did not play any role in the study or preparation of the manuscript. No conflicts of interest declared.

Keywords: cardiopulmonary resuscitation; emergency responders; out-of-hospital cardiac arrest; Vietnam

\section{Abbreviations:}

CPR: cardiopulmonary resuscitation EMS: Emergency Medical Services OHCA: out-of-hospital cardiac arrest

Received: August 22, 2021

Revised: September 27, 2021

Accepted: October 5, 2021

\section{doi:10.1017/S1049023X21001369}

(C) The Author(s), 2022. Published by Cambridge University Press on behalf of the World Association for Disaster and Emergency Medicine. This is an Open Access

\begin{abstract}
Aim: The aim of this study was to determine why bystanders did not use formal Emergency Medical Services (EMS) or conduct cardiopulmonary resuscitation (CPR) on the scene for out-of-hospital cardiac arrest (OHCA) patients in Hanoi, Vietnam.

Methods: This was a prospective, observational study of OHCA patients admitted to five tertiary hospitals in the Hanoi area from June 2018 through January 2019. The data were collected through interviews (using a structured questionnaire) with bystanders.

Results: Of the 101 patients, $79 \%$ were aged $<65$ years, $71 \%$ were men, $79 \%$ were witnessed to collapse, $36 \%$ were transported to the hospital by formal EMS, and $16 \%$ received bystander CPR at the scene. The most frequently indicated reason for not using EMS by the attendants was "using a private vehicle or taxi is faster" (85\%). The reasons bystanders did not conduct CPR at the scene included "not recognizing the ailment as cardiac arrest" (60\%), "not knowing how to perform CPR" (33\%), and "being afraid of doing harm to patients" (7\%). Only seven percent of the bystanders had been trained in CPR.

Conclusion: The information revealed in this study provides useful information to indicate what to do to increase EMS use and CPR provision. Spreading awareness and training among community members regarding EMS roles, recognition of cardiac arrest, CPR skills, and dispatcher training to assist bystanders are crucial to improve the outcomes of $\mathrm{OHCA}$ patients in Vietnam.
\end{abstract}

Vu DH, Hoang BH, Do NS, Do GP, Dao XD, Nguyen HH, Luu QT, Nguyen LH, Nakahara S. Why bystanders did not perform cardiopulmonary resuscitation on out-of-hospital cardiac arrest patients: a multi-center study in Hanoi (Vietnam). Prehosp Disaster Med. 2022;37(1):101-105.

\section{Introduction}

Out-of-hospital cardiac arrest (OHCA) is a global health challenge with large regional variations in incidence and patient prognosis: the incidence ranges 50-110 per 100,000 people per year ${ }^{1}$ and the proportion of patients' survival-to-discharge ranges from nearly $0.0 \%$ to $16.2 \% .^{2-6}$ Particularly, low- and middle-income countries with undeveloped Emergency Medical Service (EMS) systems have shown poor patient prognoses. ${ }^{2-6}$ Improving the outcomes of $\mathrm{OHCA}$ patients requires a rapid reaction chain including bystanders, EMS, and hospitals. ${ }^{7}$ Of these, the importance of bystander cardiopulmonary resuscitation $(\mathrm{CPR})$ and of the EMS dispatcher role in facilitating and instructing bystander CPR is vital. 8,9

However, in low- and middle-income countries, OHCA patients are far less likely to receive bystander CPR than those in high-income countries.,10,11 Particularly in regions with undeveloped EMS systems, severely ill or injured patients are often brought to the hospital by non-EMS vehicles. ${ }^{2-4,12}$ These situations obviously aggravate the prognoses of patients in low- and middle-income countries.

article, distributed under the terms of the Creative Commons Attribution licence (https:// creativecommons.org/licenses/by/4.0/), which permits unrestricted re-use, distribution, and reproduction in any medium, provided the original work is properly cited. 
Barriers to performing CPR include fear of doing harm to the patient, legal concerns, and fear of infections. ${ }^{13}$ Previous studies that assessed willingness to perform CPR were typically conducted in high-income countries; ${ }^{13}$ additionally, most previous studies asked participants questions about willingness and few studies asked about actual experiences. ${ }^{14}$ In low- and middle-income countries, few such studies have been conducted: a study in Lebanon indicated that lack of previous CPR training and confidence in performing CPR were negatively associated with willingness to perform CPR in OHCA patients. ${ }^{15}$

In addition to addressing the barriers to performing CPR, dispatcher assistance and instructions to bystanders is a promising measure to overcome the barriers; training dispatchers costs less than training the general population. ${ }^{8,9}$ However, in communities with low EMS use, found mostly in low- and middle-income countries, dispatcher assistance is unlikely to reach the bystanders. ${ }^{2-4,12}$ The reasons people do not use EMS in a critical condition such as OHCA have rarely been investigated as countries with functioning EMS do not encounter this issue.

In Vietnam, where EMS is undeveloped and under-utilized, investigating the reasons bystanders are reluctant to call EMS and why they do not provide basic resuscitation procedures to OHCA patients can provide useful information to streamline the chain of survival to improve patient prognoses.

\section{Methods}

\section{EMS in Hanoi}

The EMS system in Hanoi, Vietnam, run by the city government, dispatches ambulance teams staffed with physicians following the French model. ${ }^{3,16}$ It has a command center in the city center and five satellite dispatch stations with 21 ambulance units, which are independent of any hospitals, to cover the population of approximately 8.5 million in the whole city. The universal threedigit phone number $1-1-5$ is used to call EMS nation-wide. The command control center is called " 115 Hanoi Emergency Center" after the phone number. Service is provided free of charge.

\section{Study Design}

This was an observational, prospective study. Data were collected from all OHCA patients admitted from June 2018 through the end of January 2019 to the emergency departments of five participating hospitals-Bach Mai Hospital, Viet Duc Hospital, Hanoi Medical University Hospital, Agriculture General Hospital, and Hanoi Heart Hospital. Medical data were extracted from patients' medical records and information on the situations where the patient collapsed was obtained from the attendants who accompanied said patients to the hospital.

The study protocol was approved by the ethics committee of the participating hospitals and Hanoi Medical University (Protocol No. 1418-19/5/2018/IRB00003121). Oral consent was obtained from attendants before collecting information (written informed consent was waived since it was not feasible to collect such information in a critical situation where a family member or friend was dying). Informed consent was waived for data extraction from the medical records and information on this study was disclosed on the Hanoi Medical University website.

\section{Participants}

The study included OHCA patients who met all of the following criteria: (1) sudden loss of consciousness occurring outside of the medical facility; (2) cardiac arrest (deep coma, apnea, pulseless in carotid artery) confirmed by medical staff (doctor) upon arrival at the hospital or by emergency medical staff at the scene (OHCA cases confirmed by EMS staff, if attained return of spontaneous circulation before hospital arrival, were included); and (3) directly transported to one of the participating hospitals.

Patients who met one of the following exclusion criteria were excluded: (1) diagnosis of OHCA not confirmed by a qualified person or medical staff; (2) cardiac arrest in an ambulance or witnessed by medical personnel on duty; (3) transferred from another medical facility; (4) patients' attendants declined participate; (5) incomplete patients' medical record data; and (6) failure to monitor patient outcomes after discharge.

\section{Data Collection}

Data were collected through interviews based on a structured questionnaire and data extraction from the medical records. The questionnaire was prepared according to Utstein-style ${ }^{17}$ to accurately collect information regarding the situation of OHCA. It also collects information about behaviors according to cultural and social environments of Hanoi. It was pilot-tested before implementation of the study. Emergency physicians (members of this study group) in each site conducted the interviews, extracted clinical data, and recorded both on a data collection form. They were trained for interviewing, data extraction, and filling out the form.

When patients were transported to the emergency department of the hospital, the attendants (family members, friends, colleagues, or witnesses) were interviewed who brought or accompanied the patients. The collected information included the situations where the patient collapsed, witnessed or not, first aid provided, the reason they did not provide CPR, the means of transporting patients to hospitals, and the reason for not using EMS when using other means.

In the hospital, the attending physician diagnosed likely causes of patients' OHCA. Patients' outcomes were assessed using the Cerebral Performance Category score at the time of discharge or treatment completion. ${ }^{17}$ Data on hospital treatment and patient outcomes were collected from medical records stored in the hospital and follow-up records.

\section{Data Analysis}

Emergency Medical Service use and provision of bystander CPR to OHCA patients, as well as the reasons bystanders did not call formal EMS or did not provide CPR to patients, were described. The necessary sample size was estimated to ensure a $95 \%$ confidence interval for $10 \%$ of the proportion of the main reason not to perform CPR, assuming that $50 \%$ of the participants did not receive bystander CPR for this reason. The calculation was based on the following formula:

$$
95 \% \text { confidence interval }=1.96 \times \sqrt{\mathrm{p}(1-\mathrm{p}) / \mathrm{n}}<0.1,
$$

where $\mathrm{p}$ is the proportion $(0.5)$ and $\mathrm{n}$ is the necessary sample size. This resulted in a required sample size of 96 . A margin of five was allowed for some participants meeting the exclusion criteria $(\mathrm{N}=101)$. Collected data were analyzed using SPSS 20.0 (IBM Corp.; Armonk, New York USA).

\section{Results}

\section{Patients' Characteristics}

Of the $101 \mathrm{OHCA}$ patients included in this study, most were aged less than 65 years (79\%), men (71\%), collapsed at home (49\%) or on the road (33\%), were witnessed to collapse (79\%), and had internal 


\begin{tabular}{|c|c|}
\hline Characteristic & n (\%) \\
\hline \multicolumn{2}{|l|}{ Age (years) } \\
\hline$<15$ & $4(4.0 \%)$ \\
\hline $15-44$ & $42(41.5 \%)$ \\
\hline $45-64$ & $34(33.7 \%)$ \\
\hline$\geq 65$ & $21(20.8 \%)$ \\
\hline \multicolumn{2}{|l|}{ Sex } \\
\hline Female & $29(28.7 \%)$ \\
\hline Male & $72(71.3 \%)$ \\
\hline \multicolumn{2}{|l|}{ Location } \\
\hline Home & $49(48.5 \%)$ \\
\hline On the Road & $33(32.7 \%)$ \\
\hline Workplace & $4(4.0 \%)$ \\
\hline Sport Centre & $2(2.0 \%)$ \\
\hline Other & $13(12.9 \%)$ \\
\hline Witnessed & $80(79.2 \%)$ \\
\hline \multicolumn{2}{|l|}{ Who Witnessed $(n=80)^{a}$} \\
\hline Relative & $45(56.3 \%)$ \\
\hline Passer-By & $20(25.0 \%)$ \\
\hline Colleague/Friends & $13(16.3 \%)$ \\
\hline Other & $2(2.5 \%)$ \\
\hline \multicolumn{2}{|l|}{ Identified Manifestations } \\
\hline Coma & $97(96.0 \%)$ \\
\hline Apnea & $64(63.4 \%)$ \\
\hline Pulseless & $7(6.9 \%)$ \\
\hline Fall Down & $8(7.9 \%)$ \\
\hline Convulsion & $1(1.0 \%)$ \\
\hline Other & $4(4.0 \%)$ \\
\hline \multicolumn{2}{|l|}{ Likely Cause of Arrest } \\
\hline Cardiac Etiology & $28(27.7 \%)$ \\
\hline Non-Cardiac Etiology & $33(32.7 \%)$ \\
\hline Trauma & $33(32.7 \%)$ \\
\hline Non-Trauma External Causes & $7(8.8 \%)$ \\
\hline \multicolumn{2}{|l|}{ Prognoses } \\
\hline $\begin{array}{l}\text { ROSC Before Hospital Arrival or } \\
\text { At Hospital }\end{array}$ & $50(49.5 \%)$ \\
\hline Survive at Discharge & $9(8.9 \%)$ \\
\hline $\begin{array}{l}\text { Good Neurological Outcome } \\
\text { (CPC 1-2) }\end{array}$ & $1(1.0 \%)$ \\
\hline
\end{tabular}

Table 1. Participants' Characteristics $(\mathrm{N}=101)$

Abbreviations: CPC, Cerebral Performance Category; ROSC, return of spontaneous circulation.

${ }^{\text {a }}$ Includes witnessed cases.

causes (60\%; Table 1). Bystanders recognized coma and apnea in most patients; however, they rarely recognized pulselessness. One-half of the patients (50\%) attained return of spontaneous circulation before arriving at the hospital or emergency department. Few (9\%) were discharged alive and only few (1\%) showed good neurological functioning of Cerebral Performance Category score of one or two.

\section{Main Results}

Of the bystanders, 41 (41\%) called EMS; however, five did not wait for EMS and used a non-EMS vehicle. Thirty-six (36\%) were

\begin{tabular}{|c|c|}
\hline EMS Use/Bystander CPR & n (\%) \\
\hline Made EMS Call & $41(40.6 \%)$ \\
\hline \multicolumn{2}{|l|}{$\begin{array}{l}\text { Reasons for Not Calling EMS } \\
(n=60)^{a}\end{array}$} \\
\hline $\begin{array}{l}\text { Expected Faster Vehicle } \\
\text { (eg, Private Car or Taxi) }\end{array}$ & $51(85.0 \%)$ \\
\hline Did Not Trust EMS Quality & $1(1.7 \%)$ \\
\hline EMS Call was Unsuccessful & $1(1.7 \%)$ \\
\hline \multicolumn{2}{|l|}{ Transport Means to Hospital } \\
\hline Formal EMS & $36(35.6 \%)$ \\
\hline Private Car & $41(40.6 \%)$ \\
\hline Private Motorcycle & $2(2.0 \%)$ \\
\hline Taxi & $21(20.8 \%)$ \\
\hline Private Ambulance & $1(1.0 \%)$ \\
\hline $\begin{array}{l}\text { Presumed Cardiac Arrest } \\
\text { Occurring on way to Hospital by } \\
\text { Non-EMS Vehicle }(n=65)^{\mathrm{b}}\end{array}$ & $7(10.8 \%)$ \\
\hline Bystander CPR Provided & $16(15.8 \%)$ \\
\hline \multicolumn{2}{|l|}{$\begin{array}{l}\begin{array}{l}\text { Reason for Not Providing CPR } \\
(\mathrm{n}=85)^{\mathrm{c}}\end{array} \\
\end{array}$} \\
\hline $\begin{array}{l}\text { Did Not Recognize Ailment as } \\
\text { Cardiac Arrest }\end{array}$ & $51(60.0 \%)$ \\
\hline Did Not Know CPR Procedures & $28(32.9 \%)$ \\
\hline Fear of Doing Harm to the Patient & $6(7.1 \%)$ \\
\hline Having Received CPR Training & $7(6.9 \%)$ \\
\hline Dispatcher Assistance for CPR & $0(0.0 \%)$ \\
\hline
\end{tabular}

Table 2. EMS Use, Provision of Bystander CPR, and Reasons for Not Doing Them $(\mathrm{N}=101)$

Abbreviations: CPR, cardiopulmonary resuscitation; EMS, Emergency Medical Services.

${ }^{a}$ The denominator of the percentages is those whose bystander did not call EMS $(n=60)$.

${ }^{\mathrm{b}}$ The denominator of the percentage is those who were transported by non-EMS vehicles $(n=65)$. Collapse with loss of consciousness, respiration, and pulses were presumed to be cardiac arrest.

${ }^{c}$ The denominator of the percentages is those who did not receive bystander CPR $(n=85)$.

transported by EMS (Table 2). Non-EMS vehicles were mostly private cars and taxis. Of the patients transported by non-EMS $(n=65), 11.0 \%$ experienced presumed cardiac arrest (they collapsed with loss of consciousness, respiration, and pulses) on the way to the hospital. Among those who did not call EMS $(n=60)$, the most frequently indicated reason was "using a private vehicle or taxi is faster" (85\%). Among all patients, 50\% (95\% confidence interval, 40.8\%-60.2\%) used non-EMS vehicles because they were considered faster than EMS $(n=51)$.

Most patients (84\%) did not receive bystander chest compression while coming to the hospital or waiting for EMS (Table 2). Of the bystanders who did not provide CPR $(n=85)$, most (60\%) indicated the reason as "not recognizing cardiac arrest," followed by "not knowing how to perform CPR" (33\%), and a few (7\%) indicated "fear of doing harm" as the reason. Among all patients, one-half did not receive bystander CPR because cardiac arrest was not recognized $(n=51 ; 50.0 \%$; 95\% confidence interval, 40.8\%-60.2\%). Only a few bystanders (7\%) had received CPR training. There was no dispatcher-assisted CPR for those referred to EMS. 


\section{Discussion}

This study showed the ineffectiveness of the two links in the chain of survival in Hanoi: most OHCA patients were transported to the hospital without EMS being contacted and without receiving bystander CPR. Those who witnessed cardiac arrest or accompanied the patients decided not to use EMS mainly because they believed that using other means such as private vehicles or taxis was faster than using EMS. The main reason for not providing basic CPR to the patients was not recognizing the ailment as cardiac arrest rather than fear of doing harm or litigation, which have previously been reported as barriers to performing CPR. ${ }^{13-15}$ To the authors' knowledge, this was the first study to explore the reasons EMS were not utilized for cardiac arrest patients in Hanoi.

\section{EMS Use}

Bystanders' decision not to use EMS seemed rational in view of the fastest arrival to the hospital in traffic situations in central Hanoi. People have easy access to private vehicles (eg, taxis and private cars) and traffic congestion may delay EMS arrival. The average response time of EMS in Hanoi is approximately 15 minutes, which is much longer than that in other countries. ${ }^{5,12}$ If the family members or colleagues are already transporting a patient by private car, sudden collapse of the patient would naturally force them to rush to the hospital instead of stopping to call EMS.

Focusing on the travel time to hospital when deciding whether to use EMS may reflect people's perceptions of EMS as a transport service. Although fast transportation of patients to hospitals plays an important role in EMS, it has various functions that start at the beginning of the call to EMS. The EMS dispatchers should collect information on patients' condition and provide appropriate advice concerning first aid, which can facilitate bystander CPR in the case of OHCA patients. ${ }^{8,9}$ During transportation, EMS should provide Advanced Life Support care, including CPR and drug administration. These are important EMS functions that should be known to the public; however, they are not fully implemented in Hanoi.

The Hanoi EMS has resource limitations that deter the improvement of its responses. ${ }^{16}$ It covers the entire population of approximately 8.5 million in Hanoi City with 21 ambulance units. This means one ambulance unit should cover more than 400,000 population. Although hospitals in Hanoi have their own ambulances, such vehicles are used only for inter-hospital referral and are not mobilized for prehospital care.

Limited EMS functions are a problem in low- and middleincome countries because transporting severely ill or injured patients without appropriate prehospital care by EMS personnel could deteriorate patients' prognoses. ${ }^{12} \mathrm{~A}$ study in Pakistan showed similar results owing to limited EMS resources: more than one-half of the OHCA patients came to the hospital by non-EMS vehicles. ${ }^{4}$ Patients utilizing non-EMS transportation reached the hospital earlier, with a median time of 23 minutes compared to patients utilizing EMS (median time $=30$ minutes). It seems that bystanders chose the transport mode based on shorter travel time to hospital. The overall survival to discharge was only $1.6 \%$, which decreased to $0.0 \%$ at two months after discharge.

\section{Bystander CPR}

Bystanders who did not provide chest compression cited two vital reasons: not recognizing cardiac arrest and not having CPR skills. The former indicates the importance of training lay people and dispatchers in identifying cardiac arrest. Since identifying cardiac arrest is difficult for lay people, they should be trained to start CPR upon noticing unusual breathing, including agonal gasping. Additionally, encouraging dispatcher-assisted CPR is a promising strategy, $8,9,18$ which is unfortunately non-existent in Hanoi; therefore, dispatcher training and public relations should be prioritized in Hanoi. Dispatchers should identify cardiac arrest based on information from callers and provide appropriate instructions for chest compression over the call. People should also be educated about EMS' functions.

Only after recognizing cardiac arrest can other factors, often cited in previous studies, deter bystanders from providing CPR to OHCA patients. Among those who recognized cardiac arrest in this study, lack of CPR skills was the main barrier. Unlike studies in other countries, few people indicated fear of doing harm and no participants indicated fear of litigation or infection as the reason. ${ }^{13,15}$ This result reflects the fact that few CPR training opportunities exist in Hanoi. ${ }^{19}$ In Vietnam, CPR is a mandatory training program only in medical colleges; there are very few $\mathrm{CPR}$ training programs in the community and schools. Even for medical staff, unless they work in the emergency department, training and regular updating of CPR are rarely performed. Therefore, the number of people who know about cardiac arrest and have CPR skills in Hanoi is limited.

In developed countries, CPR training in the community has been practiced for a long time in many forms and at various levels. In most US states, CPR training is mandatory in high schools. ${ }^{20}$ In 2005 in Denmark, CPR training became mandatory by the time students graduated from middle school; bystander CPR increased following several national initiatives to improve resuscitative efforts from bystanders, including the distribution of CPR training kits to school children. ${ }^{21-23}$ Training for CPR in low- and middle-income countries has also brought many positive results. ${ }^{24}$ In Thailand, the National Institute for Emergency Medicine-the autonomous government agency organizing EMS system-has advocated community-level first-responder training programs which target village health volunteers, police officers, schoolteachers, and community leaders who are likely to encounter severely ill or injured people and are willing to help in such situations. ${ }^{25}$ First-responder training to primary health care personnel, such as community health workers, can effectively increase the number of people with skills and willingness to provide first aid to OHCA patients in the communities.

In Vietnam, there is no formal community-based CPR training program; although, a few training efforts exist. Training courses in CPR for dental clinic staff have been conducted in Vietnam. ${ }^{19}$ So far, more than 30 courses have been conducted with 1,500 trainees. These initial efforts need to be replicated in the community to improve the current situation. The trained dental staff could be potential trainers in future training programs.

This study revealed weak links in the chain of survival in Hanoi: a small proportion of OHCA bystanders contacted EMS and provided chest compression, which resulted in poor outcomes among patients. Bystanders' behaviors mainly resulted from lack of knowledge about CPR procedures and EMS roles. To improve patient prognoses, these weak links need to be addressed. First, awareness should be raised about cardiac arrest and proceeding actions to be taken in such situations (ie, contacting EMS and providing CPR). To achieve this goal, CPR training courses in the communities should be increased. Second, dispatcher training is crucial to improve their ability to identify cardiac arrest and guide the witness to perform CPR over the phone. 


\section{Limitations}

This study had several limitations. First, participants were derived from five tertiary-level hospitals in central Hanoi, which is not representative of all OHCA patients in the city. However, most severely ill or injured patients are transported to these hospitals, and this limitation did not distort the findings. Further, some OHCA patients from suburban areas may have been transported to nearby district hospitals; EMS coverage in such areas is much worse than in the central area. Second, limited information was obtained from the bystanders to avoid burdening them; however, this could reveal modifiable factors to improve Hanoi EMS.

\section{Conclusions}

This study revealed weak links in the chain of survival in Hanoi: a small proportion of OHCA patients received prehospital care by EMS and bystander CPR because bystanders did not have the knowledge of EMS functions or skills to recognize cardiac arrest and provide CPR. Major issues like these should be addressed through awareness raising, CPR training to the general public, and improving dispatcher skills.

\section{Author Contributions}

$\mathrm{BHH}$ and SN conceived the idea; BHH, DHV, GPD, HNH, TLQ and HTN collected information and drafted the manuscript; and $\mathrm{BHH}, \mathrm{DHV}$, and $\mathrm{SN}$ critically revised and rewrote the manuscript.

\section{Acknowledgements}

The authors thank Editage (www.editage.com) for English language editing.

\section{References}

1. Berdowski J, Berg RA, Tijssen JG, Koster RW. Global incidences of out-of-hospital cardiac arrest and survival rates: systematic review of 67 prospective studies. Resuscitation. 2010;81(11):1479-1487.

2. Do SN, Luong CQ, Pham DT, et al. Survival after out-of-hospital cardiac arrest, Viet Nam: multi-center prospective cohort study. Bull World Health Organ. 2021;99:50-61.

3. Hoang BH, Do NS, Vu DH, et al. Outcomes for out-of-hospital cardiac arrest transported to emergency departments in Hanoi, Vietnam: a multi-center observational study. Emerg Med Australas. 2021;33:541-546.

4. Mawani M, Kadir MM, Azam I, et al. Epidemiology and outcomes of out-of-hospital cardiac arrest in a developing country-a multicenter cohort study. BMC Emerg Med. 2016;16(1):28.

5. Ong ME, Shin SD, De Souza NN, et al. Outcomes for out-of-hospital cardiac arrests across 7 countries in Asia: the Pan Asian Resuscitation Outcomes Study (PAROS). Resuscitation. 2015;96:100-8.

6. Yan S, Gan Y, Jiang N, et al. The global survival rate among adult out-of-hospital cardiac arrest patients who received cardiopulmonary resuscitation: a systematic review and meta-analysis. Crit Care. 2020;24(1):61.

7. American Heart Association. 2015 American Heart Association guidelines update for cardiopulmonary resuscitation and emergency cardiovascular care. Circulation. 2015;132(18 Suppl 2):S315-589.

8. Ong MEH, Perkins GD, Cariou A. Out-of-hospital cardiac arrest: prehospital management. Lancet. 2018;391(10124):980-988.

9. Perkins GD, Travers AH, Berg RA, et al. Part 3: adult basic life support and automated external defibrillation: 2015 international consensus on cardiopulmonary resuscitation and emergency cardiovascular care science with treatment recommendations. Resuscitation. 2015;95(16 Suppl 1):e43-69.

10. Cardiac Arrest Registry to Enhance Survival (CARES) 2017 Annual Report. Atlanta, Georgia: Woodruff Health Sciences Center; 2018.

11. Nakahara S, Tomio J, Ichikawa M, et al. Association of bystander interventions with neurologically intact survival among patients with bystander-witnessed out-of-hospital cardiac arrest in Japan. JAMA. 2015;314(3):247-254.

12. Hoang BH, Dao XD, Nakahara S, Sakamoto T. The need for improving access to emergency care through community involvement in low- and middle-income countries: a case study of cardiac arrest in Hanoi, Vietnam. Emerg Med Australas. 2018;30(6):867-869.

13. Bhanji F, Mancini ME, Sinz E, et al. Part 16: education, implementation, and teams: 2010 American Heart Association guidelines for cardiopulmonary resuscitation and emergency cardiovascular care. Circulation. 2010;122(18 Suppl 3):S920-933.

14. Malta Hansen C, Rosenkranz SM, Folke F, et al. Lay bystanders' perspectives on what facilitates cardiopulmonary resuscitation and use of automated external defibrillators in real cardiac arrests. J Am Heart Assoc. 2017;6(3):e004572.

15. Shams A, Raad M, Chams N, Chams S, Bachir R, El Sayed MJ. Community involvement in out of hospital cardiac arrest: a cross-sectional study assessing cardiopulmonary resuscitation awareness and barriers among the Lebanese youth. Med Baltim. 2016;95(43):e5091.

16. Hoang BH, Mai TH, Dinh TS, et al. Unmet need for emergency medical services in Hanoi, Vietnam. JMA J. 2021;4(3):277-280.

17. Perkins GD, Jacobs IG, Nadkarni VM, et al. Cardiac arrest and cardiopulmonary resuscitation outcome reports: Update of the Utstein Resuscitation Registry Templates for Out-of-Hospital Cardiac Arrest: a statement for healthcare professionals from a task force of the International Liaison Committee on Resuscitation (American Heart Association, European Resuscitation Council, Australian and New Zealand Council on Resuscitation, Heart and Stroke Foundation of Canada, InterAmerican Heart Foundation, Resuscitation Council of Southern Africa, Resuscitation Council of Asia); and the American Heart Association Emergency Cardiovascular Care Committee and the Council on Cardiopulmonary, Critical Care, Perioperative and Resuscitation. Circulation. 2015;132(13): 1286-1300.

18. Hallstrom AP, Cobb LA, Johnson E, Copass MK. Dispatcher assisted CPR: implementation and potential benefit. A 12-year study. Resuscitation. 2003;57(2): 123-129.

19. Hoang BH, Nakahara S, Nguyen HT. Training of potential trainers on lay-people CPR in Vietnam. Resuscitation. 2019;136:149-150.

20. Brown LE, Halperin H. CPR training in the United States: the need for a new gold standard (and the gold to create it). Circ Res. 2018;123(8):950-952.

21. Hansen CM, Lippert FK, Wissenberg M, et al. Temporal trends in coverage of historical cardiac arrests using a volunteer-based network of automated external defibrillators accessible to laypersons and emergency dispatch centers. Circulation. 2014;130(21):1859-1867.

22. Isbye DL, Rasmussen LS, Ringsted C, Lippert FK. Disseminating cardiopulmonary resuscitation training by distributing 35,000 personal manikins among school children. Circulation. 2007;116(12):1380-1385.

23. Wissenberg M, Lippert FK, Folke F, et al. Association of national initiatives to improve cardiac arrest management with rates of bystander intervention and patient survival after out-of-hospital cardiac arrest. JAMA. 2013;310(13): 1377-1384.

24. Gross A. In developing countries, does the implementation of basic cardio-pulmonary resuscitation education in the community improve patient outcomes? Theses and Graduate Projects. 385. Augsburg, Germany: Augsburg University; 2018.

25. Chaleepad S, Impool T, Lertsinudom S, Chadbunchachai W, Nakahara S. First-responder training based on existing healthcare system in Thailand. Resuscitation. 2020;148:1-2. 